Original Article

\title{
Changes in kinematic chain dynamics between calcaneal pronation/supination and shank rotation during load bearing associated with ankle position during plantar and dorsiflexion
}

\author{
Masahiro Edo, PT, $\mathrm{PhD}^{1)^{*}}$, Sumiko Yamamoto, $\mathrm{PhD}^{2)}$ \\ 1) Division of Physical Therapy, Department of Rehabilitation Sciences, Faculty of Health Care \\ Sciences, Chiba Prefectural University of Health Sciences: 645-1 Nitona-cho, Chuo-ku, Chiba-shi, \\ Chiba 260-0801, Japan \\ 2) Department of Assistive Technological Science, Majoring of Health and Medical Sciences, Graduate \\ School, International University of Health and Welfare, Japan
}

\begin{abstract}
Purpose] This study aimed to elucidate the dynamics of the kinematic chain of shank rotation accompanying calcaneal pronation/supination during weight-bearing changes associated with ankle positions during plantar/dorsiflexion and whether this biomechanical phenomenon is affected by age. [Participants and Methods] The study included 54 participants (108 legs, 25 young and 29 old participants). A 3-dimensional motion analysis system measured the calcaneal pronation/supination and the shank rotation angles during pronation/supination of the foot when upright. The kinematic chain ratio was defined as the linear regression coefficient (shank rotation angle/calcaneal pronation/supination angle). The kinematic chain ratio was measured during plantar flexion, in an intermediate position, and dorsiflexion. [Results] Significant differences in the kinematic chain ratio were related to ankle position but not age. The mean kinematic chain ratio in all participants was $0.9 \pm 0.3$ for plantar flexion, $1.0 \pm 0.2$ for an intermediate position, and $1.3 \pm 0.4$ for dorsiflexion, showing significant differences between the 3 ankle positions. [Conclusion] During dorsiflexion, the long axis of the shank and that of the subtalar joint are nearly parallel; thus, shank rotation increases (larger kinematic chain ratio). During plantar flexion, a larger angle is created; thus, shank rotation decreases (smaller kinematic chain ratio). When analyzing the kinematic chain between calcaneal pronation/supination and shank rotation, it is essential to consider the ankle position during plantar/dorsiflexion.

Key words: Kinematic chain, Ankle position, Subtalar joint
\end{abstract}

(This article was submitted Aug. 29, 2018, and was accepted Sep. 25, 2018)

\section{INTRODUCTION}

The kinematic chain between calcaneal pronation/supination and shank rotation during load bearing (KCCS) is understood as something that transforms the surface of the rotational motion between the foot and the shank, which is important in smooth motions ${ }^{1,2)}$. The dynamics of KCCS are only known to occur through the pairing of calcaneal supination with shank extorsion and calcaneal pronation with shank intorsion; the details of each pairing are unclear. Therefore, we devised methods for quantifying the dynamics of $\mathrm{KCCS}^{3)}$ and have discovered large individual differences ${ }^{4)}$, to which foot alignment contributes $^{5}$. Nawoczenski et al. ${ }^{6}$ studied the dynamics of KCCS that occur when one moves on foot and demonstrated dynamics in which the ratio between calcaneal pronation/supination and shank rotation changes while upright. Based on this report, since moving on foot is a motion in which the ankle angle greatly changes during plantar and dorsal flexion, it is

*Corresponding author. Masahiro Edo (E-mail: masahiro.edo_53@cpuhs.ac.jp)

(C2018 The Society of Physical Therapy Science. Published by IPEC Inc.

(c) (1) $\odot$ This is an open-access article distributed under the terms of the Creative Commons Attribution Non-Commercial No DerivaCC BY NC ND tives (by-nc-nd) License. (CC-BY-NC-ND 4.0: https://creativecommons.org/licenses/by-nc-nd/4.0/) 
possible that the KCCS dynamics change due to ankle position during plantar and dorsal flexion.

If KCCS was affected by ankle position during plantar and dorsal flexion, then, as described above, performance in terms of transforming the rotational motion surface between the foot and the shank would change with position, which may help explain the mechanism of leg motion during movement. It is also desirable to clarify that this change in KCCS dynamics due to ankle position is not affected by age to increase our understanding of in terms of kinematic properties of the foot. Therefore, here we aimed to understand changes in KCCS dynamics due to ankle position during plantar and dorsal flexion as well as investigate whether they were affected by age.

The kinematic terminology used to refer to the foot in this article conforms to the definitions of the International Society of Biomechanics ${ }^{7}$, and supination/pronation refer to movement on the frontal plane.

\section{PARTICIPANTS AND METHODS}

The study included a total of 54 participants (108 legs), comprised of 25 young people (50 legs) (young group) and 29 elderly people (58 legs) (elderly group) with no previous history involving the lower limbs. The young group was comprised of 17 men and eight women aged $26.4 \pm 3.7$ years, measuring $167.4 \pm 8.3 \mathrm{~cm}$ in height and $59.0 \pm 9.2 \mathrm{~kg}$ in weight. The elderly group was comprised of 15 men and 14 women aged $73.5 \pm 3.3$ years, measuring $156.7 \pm 7.6 \mathrm{~cm}$ in height and 54.2 $\pm 9.2 \mathrm{~kg}$ in weight. This study was performed after obtaining approval from the ethics review committee of International University of Health and Welfare (approval number: 11-156).

The method used to quantify kinematic coupling behavior was the method described below, the validity and reproducibility of which was confirmed in an authors' previous study ${ }^{3}$. The position of infrared light-reflecting markers attached to the lower limbs of participants was measured using an optical 3-dimensional motion analysis system (Vicon Motion Systems, Oxford, UK). Markers were attached to the fibular head, medial tibial condyle, medial malleolus, lateral malleolus, posterior surface of the heel, medial surface of the heel, lateral surface of the heel, head of the first metatarsal, and head of the fifth metatarsal on both feet. Measurements were taken during active pronation/supination of both feet in a standing position. Starting from a comfortable standing position, participants were made to supinate both their feet simultaneously to a maximally supinated position, then pronate both their feet to a maximally pronated position, then supinate their feet again to a maximally supinated position, and repeat this exercise 6 times. A local coordinate system of the calcaneus, shank, and foot was created based on the positional data obtained from the reflective markers using the "BodyLanguage" programming language. Then, angle of calcaneal pronation/supination relative to the shank and angle of shank rotation relative to the foot were calculated using Euler angle. The linear regression coefficient for the angle of calcaneal pronation-to-supination and angle of shank rotation was defined as the kinematic chain ratio (KCR) and was used as an indicator of kinematic coupling behavior. The KCR is the ratio of angular variation during shank rotation relative to calcaneal pronation-to-supination (angle of shank rotation/angle of calcaneal protonation-to-supination), where the larger the KCR the greater kinematics is dominated by shank rotation, and the smaller the KCR the greater kinematics is dominated by calcaneal pronation/supination.

In this study, to better understand the changes in KCR due to ankle position during plantar and dorsal flexion in addition to the position used for the dynamic measurement method described above, we also measured the ankle in plantar flexion and dorsal flexion. That is, we measured KCCS in three positions, namely, the relaxed upright position (intermediate position); support of the body with a stand placed in front or behind, a position in which the body was tilted forward so that the ankle had a dorsal flexion of approximately $15^{\circ}$ (dorsal flexion position); and a position in which the body was tilted backward for plantar flexion of approximately $15^{\circ}$ (plantar flexion position). Accordingly, we obtained the KCR for the intermediate, dorsal flexion, and plantar flexion positions in addition to the average ankle angle during plantar and dorsal flexion (a positive value indicates plantar flexion).

We confirmed the normality of the data using the Shapiro-Wilk test and used repeated-measures two-way analysis of variance of the KCR differences using ankle position during plantar and dorsal flexion and age as the factors and Tukey's honestly significant differences test for the post-hoc comparison. Left and right leg data were handled independently during analysis, and the significance level used in tests was a hazard ratio of $5 \%(\mathrm{p}<0.05)$. Statistical analysis was performed using IBM SPSS Statistics 25 (IBM Co., Armonk, NY, USA) statistical analysis software.

\section{RESULTS}

The measurement result of KCR in the representative example is shown in Fig. 1.

Regarding differences in KCR, using the ankle position during plantar or dorsal flexion and age as the factors, significant differences were only found for ankle position, while differences in age and interactions were not significant. The average KCR for all participants was $0.90 \pm 0.29$ for plantar flexion, $1.00 \pm 0.23$ for intermediate, and $1.31 \pm 0.38$ for dorsal flexion; significant differences were found among all positions (Table 1). The average plantar flexion angle of the ankle during measurements was $18.63^{\circ} \pm 4.97^{\circ}$ for plantar flexion, $-0.07^{\circ} \pm 2.81^{\circ}$ for intermediate, and $-13.15^{\circ} \pm 4.33^{\circ}$ for dorsal flexion. 


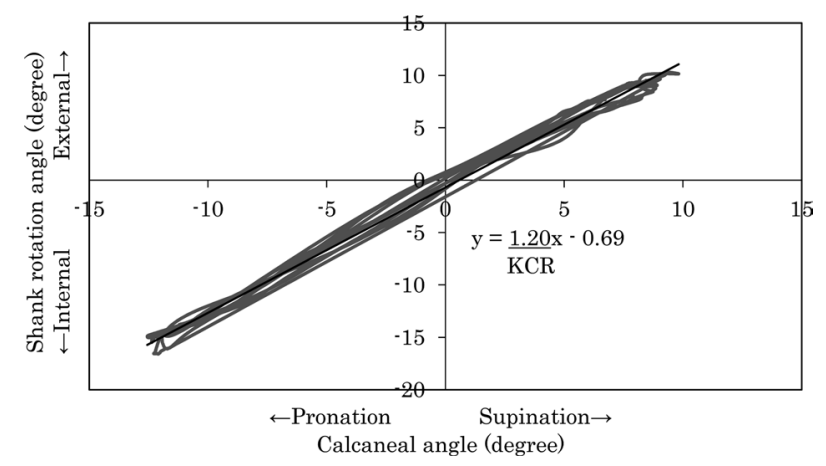

Fig. 1. Indicators definition of behavior of KCCS (KCR) (Representative example).

Table 1. KCR differences by ankle position and age

\begin{tabular}{llccc}
\hline & & \multicolumn{3}{c}{ Ankle position } \\
\cline { 3 - 5 } & & Plantar flexion & Intermediate & Dorsal flexion \\
\hline \multirow{2}{*}{ Age } & Young group & $0.83 \pm 0.26$ & $1.00 \pm 0.22$ & $1.32 \pm 0.43$ \\
& Elderly group & $0.95 \pm 0.31$ & $1.00 \pm 0.24$ & $1.30 \pm 0.34$ \\
Mean & & $0.90 \pm 0.29$ & $1.00 \pm 0.23$ & $1.31 \pm 0.38$ \\
\hline
\end{tabular}

2 way ANOVA (ankle position: ***, age: n.s., ankle position $\times$ age: $n . s$.)

Tukey's HSD test (plantar flexion vs. intermediate: *, plantar flexion vs. dorsal flexion: ***, intermediate vs. dorsal flexion: ***)

$* \mathrm{p}<0.05,{ }^{* * *} \mathrm{p}<0.001$.

\section{DISCUSSION}

This study revealed that KCR magnitude decreases from dorsal flexion to intermediate to plantar flexion. Accordingly, it has been suggested that, in KCCS dynamics, shank rotation degree increases as ankle position changes from plantar flexion to dorsal flexion. Also, no effect of age was found in this change of KCR due to ankle position during plantar and dorsal flexion.

Our earlier study of the relationship between $\mathrm{KCR}$ and foot bone alignment ${ }^{5)}$ found that $\mathrm{KCR}$ increases as the joint surface of the subtalar joint tilts further backward; therefore, it is possible that the direction of the subtalar joint axis contributes to this change. The axis of the subtalar joint moves from the backward and downward direction toward the forward and upward direction in the sagittal plane ${ }^{8}$, so it can be hypothesized that the axis of motion due to backward tilting of the joint surface tends to cause shank rotation since it is nearly parallel to the long shank axis and that the axis of motion due to forward tilting of the joint surface tends to cause calcaneal pronation and supination since it is nearly parallel to the long foot axis. Thus, we hypothesize that the reason that the present study found that large shank rotation occurs in ankle dorsal flexion, that is, the KCR is large, is that the long shank axis is nearly parallel to the subtalar joint axis. Conversely, the KCR was small in ankle plantar flexion because the long shank axis creates a large angle with the subtalar joint axis.

The KCCS functions while moving on foot are thought to be to absorb the rotational stress on the leg by converting it to foot pronation and supination ${ }^{1)}$ and transmit calcaneal pronation and supination as a trigger of rotational motion to the leg ${ }^{2}$. We infer that it is better when these functions use the dorsal flexion position, in which KCR is large, because it is desirable that shank rotation be greater than calcaneal pronation and supination during such functions. Accordingly, one should verify that, when shank rotation is controlled by calcaneal pronation and supination such as due to the foot plate, the ankle is in dorsal flexion. Many researchers have said that KCCS functions are important ${ }^{1,2,6,9)}$; in the future, it will be necessary to consider that the dynamics change with ankle position.

\section{Conflict of interest}

None. 


\section{REFERENCES}

1) Morris JM: Biomechanics of the foot and ankle. Clin Orthop Relat Res, 1977, (122): 10-17. [Medline]

2) Nicholas JA, Hershman EB: The lower extremity and spine in sports medicine. St. Louis: Mosby, 1986, pp 395-411.

3) Edo M, Yamamoto S, Yonezawa T: Validity and reproducibility of measuring the kinematic coupling behavior of calcaneal prona-tion/supination and shank rotation during weight bearing using an optical three-dimensional motion analysis system. Int J Physiother, 2017, 4: 343-347. [CrossRef]

4) Edo M, Yamamoto S: Differences in kinematic coupling behavior of calcaneal pronation/supination and shank rotation during weight bearing based on age, sex, and laterality: analysis of kinematics using optical three-dimensional motion analysis system. Int J Physiother, 2018, 5: 31-35. [CrossRef]

5) Edo M, Yamamoto S, Yonezawa T: Factors that determine kinematic coupling behavior of calcaneal pronation/supination and shank rotation during weight bearing: an analysis based on foot bone alignment using radiographic images. J Phys Ther Sci, 2018, 30: 1215-1220. [CrossRef]

6) Nawoczenski DA, Saltzman CL, Cook TM: The effect of foot structure on the three-dimensional kinematic coupling behavior of the leg and rear foot. Phys Ther, 1998, 78: 404-416. [Medline] [CrossRef]

7) Wu G, Siegler S, Allard P, et al. Standardization and Terminology Committee of the International Society of Biomechanics International Society of Biomechanics: ISB recommendation on definitions of joint coordinate system of various joints for the reporting of human joint motion-part I: ankle, hip, and spine. J Biomech, 2002, 35: 543-548. [Medline] [CrossRef]

8) Lundberg A, Svensson OK, Nemeth G, et al.: The axes of rotation of the talocalcaneal and talonavicular joints. Foot, 1993, 3: 65-70. [CrossRef]

9) Pinto RZ, Souza TR, Trede RG, et al.: Bilateral and unilateral increases in calcaneal eversion affect pelvic alignment in standing position. Man Ther, 2008, 13: 513-519. [Medline] [CrossRef] 